\title{
The Harmonic Scalpel
}

\author{
Dilip Kumar Dutta ${ }^{1} \cdot$ Indranil Dutta $^{1}$
}

Published online: 26 February 2016

(C) Federation of Obstetric \& Gynecological Societies of India 2016

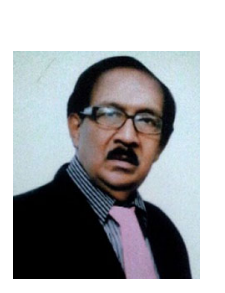

\section{About the Reviewer}

Dr. Dilip Kumar Dutta is currently Chairman ICOG, FOGSI 2015. He is an author of 30 Dutta's Books. He is Medical Director of GICE Hospital, Kalyani, West Bengal. He has presented over 400 papers in nationally and over 45 papers internationally. He has over 80 article publication in various journals.

Dr. Dilip Kumar Dutta M.D, FRCOG, Ph.D, MAMS, FICOG, FICMCH, FIAMS, is currently Chairman in Medical Director at GICE Hospital; Indranil Dutta is the Assistant Professor in IQCMC Medical College.

$\triangle$ Dilip Kumar Dutta

drdilipdutta@yahoo.com

1 Kalyani, India
Any laparoscopic surgery demands appropriate surgical instruments. Most surgeons nowadays have adapted to this different form of surgery and have particular preferences for certain types of graspers, dissectors and cutting instruments. The general component of laparoscopic instrument includes the hand grip, shaft, jaw and the tip.

The harmonic scalpel is a surgical instrument used to simultaneously cut and cauterize tissue. It is the latest device to have been introduced in last decade or so. 
Ultrasonic energy is famously used in Harmonic scalpel where ultrasonic energy is converted to mechanical energy at the active blade [1]. The main mechanism is the active blade which delivers high-grade frictional force while the inactive upper arm holds tissue in apposition. Its main advantages include precise dissection, reliable hemostasis, less lateral thermal spread and charring mainly works by applying pressure and then sealing with a denatured protein coagulum while applying ultrasonic vibration to denature hydrogen bonds perform vessel coagulation [2]. Ultrasicon is the name given to the dissection method by harmonic. The transducer in the handpiece consisted of piezoelectric crystal sandwiched under pressure among metal cylinders. The ultrasonic generator converts ultrasonic energy into mechanical energy. The sealing of the vessels is achieved due to denatured protein coagulum which occurs due to tamponade and coaptation. It has three compatible probes that are the shear, blade and a hook. The shear can coagulate vessels up to $5 \mathrm{~mm}$, whereas the hook and blade only $2 \mathrm{~mm}$ in diameter. It has the least thermal spread and smoke production of all the devices. Ethicon first introduced the world to HARMONIC ultrasonic technology and its precise dissection capability in 1998 with the release of the HARMONIC ${ }^{\circledR}$ Scalpel Shears. Manufactured and supplied by ETHICON a part of Johnson and Johnson, it is a wonderful device available in various types. The latest one of the harmonic devices is HARMONIC $\mathrm{ACE}^{\circledR}+7$ Shears with advanced hemostasis.

It is one of the first devices with purely ultrasonic energy device with a 7-mm vessel sealing indication. It is known to have advanced adaptive tissue technology which delivers unmatched precision, multifunctionality, and now advanced hemostasis for stronger large vessel sealing. It ensures less tissue damage and it is claimed to be stronger, more reliable sealing device and is successful in being able to seal vessels with $7 \mathrm{~mm}$ diameter. It has higher median burst pressure versus LigaSure ${ }^{\mathrm{TM}} 5$-mm blunt tip, when sealing 5-7-mm vessels in the advanced hemostasis mode. Harmonic also has some credible reports, suggesting that it has better results in term of less postoperative pain complains by patient [3]. The development of ultrasonically activated coagulating shears in the early 1990s has provided an alternative to other methods of controlling blood vessels. The device divides tissue by using high-frequency $(55,000 \mathrm{~Hz})$ ultrasonic energy transmitted between the instrument blades. The active blade of the instrument vibrates longitudinally against an inactive blade over an excursion of 50-100 $\mu \mathrm{m}$. Hence, this wonder device is still much in demand but cost can be a factor for every surgeon, otherwise an marvel of device.

\section{Newer Devices}

THUNDERBEAT ${ }^{\circledR}$ (Olympus Medical Systems Corp., Tokyo, Japan), with a conventional ultrasonic scissor and a bipolar vessel clamp, with respect to safety (thermal profile and histologic damage) and efficacy (sealing capability and cutting speed), was used. The versatility of THUNDERBEAT was based on the following five points: hemostasis, histologic sealing, cutting, dissection and tissue manipulation. The main benefits included the following: 1. fastest in class cutting speed, thereby reducing operation time; 2 . reliable 7-mm vessel sealing; 3 . precise dissection with fine jaw design; 4. always available bipolar energy for hemostasis without cutting; 5. minimal thermal spread; 6 . fewer instrument exchanges; 7. reduced mists generation help to maintain visibility.

\section{Compliance with Ethical Standards}

Conflict of interest Indranil Dutta, Dilip Kumar Dutta declare that they have no conflicts of interest.

Ethical Statement All procedure followed were in accordance with the ethical standards of the responsible committee on human experimentation (institutional and national) and with the Helsinki Declaration of 1975, as revised in 2008 .

\section{References}

1. Seehofer D, Mogl M, Boas-Knoop S, et al. Safety and efficacy of new integrated bipolar and ultrasonic scissors compared to conventional laparoscopic 5-mm sealing and cutting instruments. Surg Endosc. 2012;26(9):2541-9.

2. Lenihan J, Kovanda C, Commerano C. Comparison of laparoscopic-assisted vaginal hysterectomy with traditional hysterectomy for cost-effectiveness to employers. Am J Obstet Gynecol. 2004;190:1714-22.

3. Litta P, Fantinato S, Calonaci F, et al. A randomized controlled study comparing harmonic versus electrosurgery in laparoscopic myomectomy. Fertil Steril. 2010;94:1882-6. doi:10.1016/j. fertnstert.2009.08.049. 